\title{
Recent advances in capillary electrophoresis instrumentation for the analysis of explosives
}

\author{
Matías Calcerrada a , Miguel González-Herráez b , Carmen García-Ruiz a,** \\ a Department of Analytical Chemistry, Physical Chemistry and Chemical Engineering, Multipurpose Building of Chemistry, University of Alcalá, Ctra. \\ Madrid-Barcelona km 33.600, 28871 Alcalá de Henares (Madrid), Spain \\ b Departament of Electronics, Politechnic School, University of Alcalá, Ctra. Madrid-Barcelona km 33.600, 28871 Alcalá de Henares (Madrid), Spain
}

\section{A R T I C L E I N F O}

\section{Keywords:}

Capillary electrophoresis

Explosive

Inorganic

Instrumentation

Microchip

Organic

Portable CE

Recent CE

Review

Sensor

\begin{abstract}
A B S T R A C T
Capillary electrophoresis (CE) is a well-established analytical separation technique. Owing to its high versatility, major advancements have been made with regard to the instrumental set-ups during the last years. New strategies have been proposed to develop high-sensitive methods, portable CE or miniaturized devices. These techniques are of great interest in the analysis of explosives, which generally requires a highly selective approach. This review provides a recent perspective (from the beginning of 2008 to March 2015) on the use of CE for the analysis of explosives. First, a general description of explosives is made, emphasizing the role of separation techniques and specifically CE. Next, the most recent works focused on the analysis of explosives by using conventional $\mathrm{CE}$, portable $\mathrm{CE}$ and microchip $\mathrm{CE}$ are compared and critically discussed. Besides, other emerging techniques for the analysis of explosives are referred and compared to CE results. Finally, future perspectives for the analysis of explosives by CE are proposed.
\end{abstract}

(C) 2015 Elsevier B.V. All rights reserved.

\section{Contents}

1. Introduction

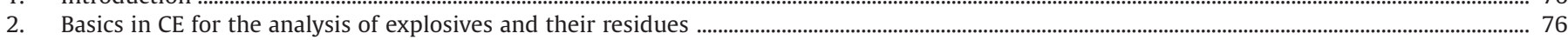

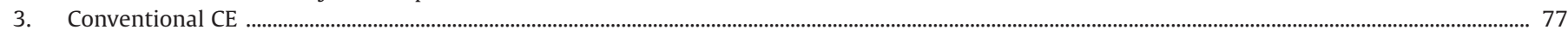

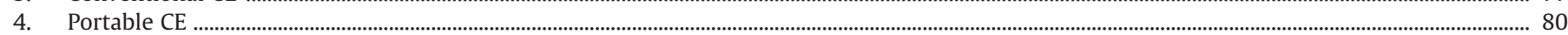

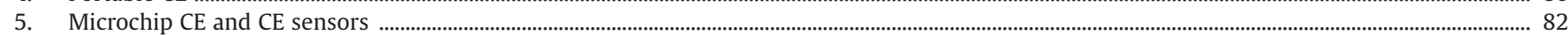

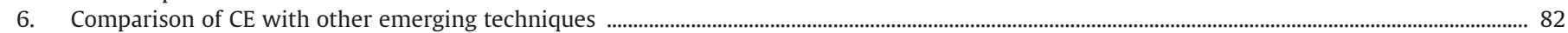

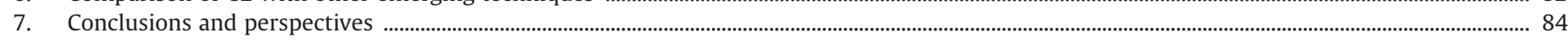

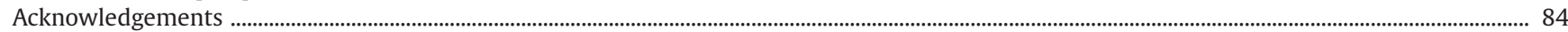

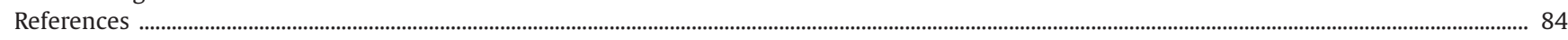

Abbreviations: AD, amperometric detection; ANAL, ammonium nitrate aluminium powder; ANFO, ammonium nitrate fuel oil; APTS, 8-aminopyrene-1,3,6-trisulfonic acid;

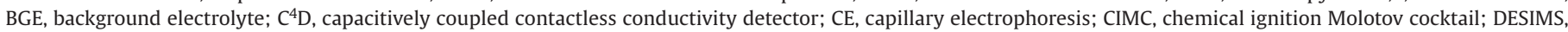

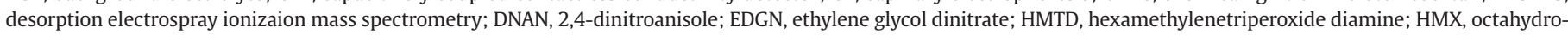

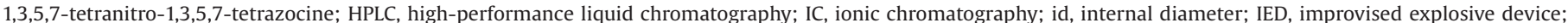

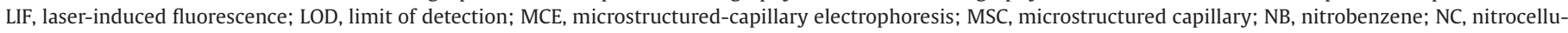

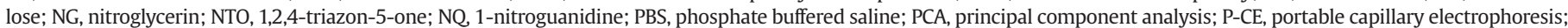

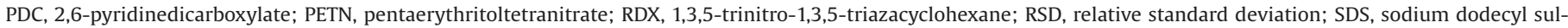

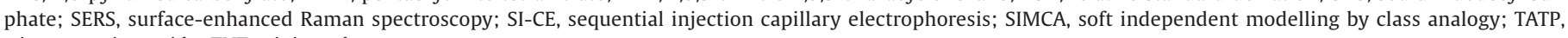
triacetonetriperoxide; TNT, trinitrotoluene.

* Corresponding author. Tel.: +34 91 8856431; Fax: +34 918854971.

E-mail address: carmen.gruiz@uah.es (C. García-Ruiz). 


\section{Introduction}

An explosive is a substance or a device that when subjected to heat, impact, friction or detonation releases a large amount of energy. This energy in the form of hot gases expands rapidly creating a pressure wave, called "shock wave". Depending on their chemical structure, use or nature, explosives have been classified at different levels. [1,2] For example, they can be divided into high-order explosives (expansion speed range: between 50,000 and 10,000 m/s) or low-order explosives (expansion speed range: between 300 and $3000 \mathrm{~m} / \mathrm{s}$ ). High-order explosives can be subcategorized into primary explosives, such as nitroglycerin (NG), hexamethylenetriperoxide diamine (HMTD) or triacetonetriperoxide (TATP), which are less stable, and thus used in small quantities in primers or detonators; secondary explosives such as pentaerythritoltetranitrate(PETN), 1,3,5trinitro-1,3,5-triazacyclohexane (RDX), octahydro-1,3,5,7-tetranitro1,3,5,7-tetrazocine (HMX), dynamite or trinitrotoluene (TNT), being more stable to mechanical shock and also more energetic; and tertiary explosives, such as ammonium nitrate fuel oil (ANFO) or ammonium nitrate aluminium powder (ANAL), the most stable explosives, requiring an intermediate explosive booster of secondary explosives to initiate. On the other hand, low-order explosives or propellants can be detonated only under extreme conditions. Black powder (made of sulphur, charcoal and potassium nitrate) and smokeless gunpowders (principally made of nitrocellulose, NC) can be categorized within this type of explosives. Black powders can be used in pyrotechnic composition of fireworks, whereas smokeless gunpowders are a part of modern ammunitions [3], although they can also be employed for the fabrication of improvised explosive devices (IEDs).

It is worth noting that, despite being explosive substances, these materials can also be classified depending on their final use, as their chemical properties lead to different applications. On the one hand, some explosives, such as PETN, RDX, HMX, C-4 or Semtex are usually used by military forces, as their availability is limited for other areas. On the other hand, other explosives such as TNT, ANAL, ANFO, dynamite or nitroglycerin are more common substances, as they are used for civil and commercial purposes, such as building demolitions, firework displays, tunnel excavations or airbag fabrication. These applications make the analysis of explosives relevant for specific fields, such as defence, environment or industry. [4]

Apart from the above-mentioned uses, explosives are unfortunately used in terrorism attacks. In fact, during the recent years, several terrorist attacks have been carried out using a wide variety of explosives. For example, TNT was used in the Bali resort bombings in 2002; dynamite in the Madrid train station bombings in 2004; PETN in different attempts to blow up aircrafts in 2001 and 2009; and black powder-based IED in the Boston bombings in 2013employed. [2] Thus, given the variety of explosives usable for terrorist attacks, it is evident that the analysis of all of them is of high relevance from a forensic standpoint.

Different approaches have been carried out to perform the analysis of the explosive samples. For example, spectroscopic techniques have been successfully applied to a wide variety of samples and caseworks; readers interested on this issue can refer to a recent review. [2] Nevertheless, traditional separation techniques are still used for the analysis of explosives. Basically, the above-mentioned techniques allow the identification and quantification of many types of species, at very low concentrations. [5,6] Despite their destructive character, these advantages make them useful techniques for the analysis of explosives. For example, one of the most established separation techniques, high-performance liquid chromatography (HPLC) is the official and regulated-by-law method for some high-order explosive analyses in specific matrices in the US [7], or IC for the analysis of low-order explosives. [6] Nevertheless, it is noteworthy that working groups specialized in the analysis of explosives have established guidelines for the analysis of intact explosives and post-blast residues. $[8,9]$ These guidelines evidence that several analytical techniques are usually required for the analysis of these samples.

In this context, other separation techniques have provided promising results for the analysis of explosives. For example, capillary electrophoresis (CE), though not well established like HPLC, is less expensive, requires less amount of solvents and samples and, in particular, presents huge versatility. [10] Some important developments during the recent years have been the microchip technology, as well as portable $\mathrm{CE}$ (P-CE) instrumentation. These techniques satisfy some of the requirements of explosive analysis, such as the need of highly selective and high- sensitive assays with potential for on-field in situ analysis to avoid transportation of the sample. In fact, different reviews devoted to the advances of $\mathrm{CE}$ in explosives can be consulted in the literature. [11-13] However, during the last years, new approaches have been developed for the analysis of explosives, and new instrumental set-ups also have been optimized and applied to these samples. Figures of merit of this recent research are of interest from a forensic standpoint, but also to note the recent development in CE instrumentation and its applicability.

This manuscript summarizes the previously published reviews and critically reviews the recent works (from the beginning of 2008 to March 2015) on the analysis of explosives using CE. [11-13] The first section describes the traditional CE analysis of explosives and its potential advantages in the field of forensics and homeland security as well as limitations. The subsequent sections focus on the critical review of the previously published studies on the analysis of explosives using different types of $\mathrm{CE}$ : conventional $\mathrm{CE}$, the recently developed P-CE, and microchip CE. The samples, the preparation methods and details on the technique and CE conditions have been summarized in a table for comparative purposes. The final section summarizes the developments in the CE technique for the analysis of explosives and also provides a brief comparison between $\mathrm{CE}$ and other emerging techniques. Conclusions and perspectives on the use of $\mathrm{CE}$ for the analysis of explosives are made.

\section{Basics in CE for the analysis of explosives and their residues}

In this section, the performance of $\mathrm{CE}$ and its ability to detect explosives has been discussed, and a comparative study of the typical analytical techniques employed at the forensic laboratories has been presented.

As stated earlier, CE is an established technique useful for the separation of macromolecules under the application of an electric field between the extremes of the capillary. This technique presents some advantages, such as its simple instrumental set-up, versatility and expensiveness compared to other separation techniques. These advantages have been exploited during the last years, and currently new portable equipment, novel CE formats or even original CE conditions have been developed to enhance the CE performance. In this review, recent studies devoted to the analysis of explosives by novel CE approaches from a forensic and homeland security standpoint are taken into consideration.

However, compared to other separation techniques, CE has its own limitations. [14] In the case of forensic applications related to the analysis of explosives, three main goals are pursued: i) increased selectivity to minimize matrix effects in the sample; ii) obtain robust equipment allowing enough precision in the results, thus providing unambiguous interpretation; and iii) enhanced sensitivity of the systems to be able to perform the detection of both bulk and trace analytes found in the sample. Sensitivity of CE is comparable to other separation techniques. LODs in the order of nanograms have been achieved in IC recently. [15,16] However, compared to other techniques such as IC [8] or HPLC [17], CE shows less 
robustness. For example, different inorganic and organic species were recently analysed by IC, obtaining precise values; the relative standard deviation (RSD) was $<2.1 \%$ considering the peak heights and $n=10$ for cations and $n=8$ for anions. [15] More recently, IC was coupled to MS for the determination of organic and inorganic anions, which provided RSD values of retention time $<0.43$ and peak areas $<25 \%$. [18] CE, In the case of CE, using a complex separation medium with additives, which sometimes are required to achieve proper separation of the analytes under detection, may affect the robustness of the system. Due to these reasons, orthogonal techniques are usually employed to confirm the results. For example, for analyzing organic explosives used in crime, HPLC is ideally used [17] to complement CE analysis. Conversely, for the analysis of inorganic explosives or their residues (ions), CE is usually complemented with validated methods based on IC, given the reported analytical performance of this technique. [8]

In the following sections, works related to the analysis of explosives by CE using new methodologies, platforms, and formats are addressed for demonstrating the high potential applications of this technique in the detection of different and small amounts of explosives (even at trace levels depending on the CE configuration and performance). However, from a forensic standpoint, recommended that this technique be complemented with other validated spectrometric techniques or be used as a hyphenated technique (e.g. CE coupled to mass spectrometry).

\section{Conventional CE}

During the last years, the use of conventional CE over other approaches is evidenced, as can be seen in Table 1. Nevertheless, compared to the previous studies [11-13], significant advancements have been made and they are discussed according to the analytes of interest. As can be seen, most of the works have focused on the analysis of cations and anions, as some of them can be considered as marker ions (after an explosion) for the potential identification of inorganic salts contained in the explosives [19-22,24-27], and only one study was devoted to the analysis of carbohydrates. [23] Apart from the analysis of high-order explosives,28 studies have also focused on environmental analysis, with only few on forensic purposes. [28,29] Finally, other studies have been carried out for the determination of intact pre-blast nitrostarch and nitrocellulose, a secondary explosive that may be present in some explosives of interest such as dynamites or home-made explosives. [30,32-35]

$\mathrm{CE}$ is a promising candidate for the analysis of cations and anions due to its ability to detect charged macromolecules at low concentrations, and thus can be used for collection post-blast residues at crime scene. However, matrix effects and contamination must be considered due to the prevalence of anions and cations in the environment. Of note, K. G. Lahoda et al. (2008) collected totally 286 different samples from 28 different cities in the US, and comprehensively evaluated the presence of explosive residues in 16 different matrices. Collection of a large number of samples from different places allowed good interpretation of results. Organic explosives were detected by gas chromatography, whereas the inorganic residues were studied by electrokinetic chromatography with UV detection, at 208 (cations) and 235 (anions) nm. The resulting electropherograms have not been shown; however, global results indicate that the potential ions found in explosive residues, such as ammonium, nitrite, perchlorate, thiocyanate and cyanate, were hardly found, whereas others such as nitrate, chloride or sulphate are more common at crime scenes, and thus they can interfere with the results and must be specially considered [19]

Sarazin et al. developed a CE method for the analysis of cations and anions in post-blast extracts from acid-aluminium burst residues, which had not been analyzed before by EKC-UV, detecting the species at $214 \mathrm{~nm}$. The method was optimized, particularly the concentration of 2,6-pyridinedicarboxylate (PDC) in the background electrolyte (BGE) that produces the chelated form of aluminium and the cation of interest, in order to avoid the formation of other chelates from non-monovalent cations such as copper, iron, calcium or zinc chelates. Results showed that no interference anions were found, and despite not being as sensitive as IC, the high concentrations found in the real samples allowed analysis of the samples using $\mathrm{CE}$, and the expected ions, chloride from the acid and aluminium (III) were detected. [20]

Sarazin et al. also optimized three different CE-UV methods for the analysis of anions, cations and carbohydrates, including the species of interest for the analysis of post-blast residues from explosives and IEDs, and carried out comprehensive studies on matrix interferences. [21-23] The method for anions sought the applicability to most of the explosive samples in order to be used by the French security forces, thus a general method was proposed. Compared to the previous methods, this technique also involved the separation and identification of the ion azide, usually present in detonators. RSDs of normalized times for the 15 studied matrices were $<0.75 \%$ in all the cases. Finally, pipe-bomb residues were analysed to quantify chloride and chlorate anions (16 and $30 \mathrm{mg} \mathrm{L}^{-1}$, respectively), which authors claimed as possible chlorate salts used in the fabrication of the explosives, and also elevated concentrations of carbonate (26 mg L $\mathrm{m}^{-1}$ ), present in explosives and also atmospheric contaminants. [21] Indirect UV at $190 \mathrm{~nm}$ was employed for cation analysis. In order to detect the cations, authors proposed a bilayer inside the capillary (with hexadimethrine bromide as the first layer and polyvinylsulfonic acid (as sodium salt) as the second layer). After optimizing the medium and adding 18-crown-6 ether to improve the separation efficiency between some cations, they were applied to the analysis of residues from explosions at cash dispensers; cations (potassium, calcium, sodium and magnesium) as well as ammonium from the explosive ammonium nitrate were detected. Residues from fireworks contained ammonium, potassium, calcium and barium, whereas firecracker residues comprised potassium, calcium, sodium and magnesium. [22] The third method was optimized to successfully separate and detect different carbohydrates (fructose, glucose, galactose and sucrose), generally after the detonation of a home-made explosive. [23] Finally, all the three methods were tested in a simulated bombing attack, where an IED made of ammonium nitrate and icing sugar was fabricated and detonated. [24] The resulting electropherograms (Fig. 1) of the residues collected after the bombing simulation show the efficiency of the CE methodologies. Besides, note that these methodologies were also compared to the previous methods developed by IC, and despite obtaining poorer limits of detection (LODs), CE was successfully applied in all the cases, providing separation with less BGE consumption and cost-effective conditions compared to IC.

C. Martín-Alberca et al. developed a qualitative method to detect potential anion markers from chemical ignition Molotov cocktails (CIMCs). After preparing 24 different CIMCs by mixing different combinations of sulphuric acid, potassium chlorate and perchlorate, sugar, petrol, kerosene, fuel, ethanol and methanol at different proportions, only 10 worked properly. UV detection was performed at $250 \mathrm{~nm}$, and various anions were detected (chlorate, perchlorate, sulphate and chloride). After observing the chlorate and perchlorate peak areas at specific times for a period of $50 \mathrm{~h}$, the authors confirmed that chlorate degraded to perchlorate over time, on the basis of the proposed reactions for CIMCs. Finally, the combination of perchlorate, chloride, chlorate and high levels of sulphate were proposed as reliable markers for CIMCs. [25,26] The authors also employed the same CE methodology to detect anions and cations in consumer fireworks: after a physical characterization of the six types of consumer fireworks studied, which authors claimed to be essential for result interpretation, they were analysed and chemically characterized. [27] 
Table 1

Recent publications focused on the analysis of explosives and related compounds by CE. ${ }^{a}$

\begin{tabular}{|c|c|c|c|c|c|c|c|}
\hline Sample (n) & Analyte & Sample preparation & CE mode & e-detection & CE conditions & Analytical performance & Ref. \\
\hline $\begin{array}{l}286 \text { Samples } \\
\text { from } 16 \\
\text { matrices } \\
\text { (28 cities) }\end{array}$ & $\begin{array}{l}\mathrm{Cl}^{-}, \mathrm{NO}_{2}^{-}, \mathrm{NO}_{3}^{-}, \mathrm{ClO}_{4}^{-}, \\
\mathrm{ClO}_{3}^{-}, \mathrm{SCN}^{-}, \mathrm{SO}_{4}^{2-}, \mathrm{OCN}^{-} \\
\mathrm{K}^{+}, \mathrm{NH}^{+}, \mathrm{Na}^{+}, \mathrm{Ca}^{2+}, \mathrm{Mg}^{2+}\end{array}$ & $\begin{array}{l}\text { Extraction }\left(1 \mathrm{~mL} \mathrm{H}_{2} \mathrm{O}\right) \\
\text { Centrifugation } \\
(8 \mathrm{~min}, 1000 \mathrm{rpm})\end{array}$ & EKC & $\begin{array}{l}\text { UV } \\
208,235 \mathrm{~nm}\end{array}$ & $\begin{array}{l}\text { BGE: } 15 \mathrm{mM} \text { HIBA, } 6 \mathrm{mM} \\
\text { imidazole, } 4 \mathrm{mM} 18-\mathrm{c}-6,3 \\
\text { mMNTS, and AcN } 5 \%(\mathrm{v} / \mathrm{v}) \text {, } \\
\text { at pH } 6.5 \\
\text { Capillary: } 40.2 \mathrm{~cm}, 50 \mu \mathrm{m} \text { id } \\
\text { Injection: } 7 / 5 \mathrm{~s} \\
\text { Separation: } 10 \mathrm{kV}, 25^{\circ} \mathrm{C}\end{array}$ & $\begin{array}{l}\text { LOD: } 1-15 \mathrm{mg} \mathrm{L}^{-1} \\
\mathrm{SD}_{\text {tmig }}<0.013 \\
\text { (cations), } 0.035 \\
\text { (anions) }\end{array}$ & [19] \\
\hline $\begin{array}{l}\text { Post-blast } \\
\text { samples from } \\
\text { acid- } \\
\text { aluminium } \\
\text { mixtures }\end{array}$ & $\mathrm{Cl}^{-}, \mathrm{NO}^{3-}, \mathrm{Al}^{3+}$ & $\begin{array}{l}\text { Solution }\left(\mathrm{H}_{2} \mathrm{O}\right) / \\
\text { sonication }\left(\mathrm{H}_{2} \mathrm{O} \text {, hot }\right) \\
10 \text { min }\end{array}$ & CZE & $\begin{array}{l}\text { UV } \\
254 \mathrm{~nm}\end{array}$ & $\begin{array}{l}\text { BGE: } 10 \mathrm{mM} \text { PDC, at pH } 4.5 \\
\text { Capillary: } 60 \mathrm{~cm}, 50 \mu \mathrm{m} \text { id } \\
\text { Injection: } 0.4 \mathrm{psi}, 15 \mathrm{~s} \\
\text { Separation: } 30 \mathrm{kV}, 25^{\circ} \mathrm{C}\end{array}$ & $\begin{array}{l}\text { Linear range: } \\
1-30 \mathrm{mg} \mathrm{L}^{-1} \\
\text { LOD: } 0.3-1.2 \mathrm{mg} \mathrm{L}^{-1} \\
\text { LOQ: } 1-4 \mathrm{mg} \mathrm{L}^{-1} \\
\mathrm{RSD}_{\text {tmig }}<2.5(n=9) \\
\mathrm{RSD}_{\text {Area }}<3.9(n=9)\end{array}$ & [20] \\
\hline $\begin{array}{l}\text { Post blast } \\
\text { samples } \\
\text { (pipe-bomb) } \\
\text { from } 15 \\
\text { matrices. }\end{array}$ & $\begin{array}{l}\mathrm{CH}_{3} \mathrm{COO}^{-}, \mathrm{C}_{7} \mathrm{H}_{5} \mathrm{O}_{2}^{-}, \mathrm{CO}_{3}^{2-} \\
\mathrm{ClO}_{2}{ }^{-}, \mathrm{F}^{-}, \mathrm{S}_{2} \mathrm{O}_{3}{ }^{2}, \mathrm{PO}_{4}^{3-}, \mathrm{Cl}^{-} \\
\mathrm{NO}_{2}^{-}, \mathrm{NO}_{3}^{-}, \mathrm{ClO}_{4}^{-}, \mathrm{ClO}_{3^{-}} \\
\mathrm{SCN}^{-}, \mathrm{SO}_{4}^{2-}, \mathrm{OCN}^{-}, \mathrm{I}^{-}, \mathrm{N}_{3^{-}} \\
\mathrm{C}_{2} \mathrm{O}_{4}{ }^{2-}\end{array}$ & $\begin{array}{l}\text { Extraction }\left(\mathrm{H}_{2} \mathrm{O} \text {, hot }\right) \text {, } \\
\text { sonication }(10 \mathrm{~min})\end{array}$ & CZE & $\begin{array}{l}\text { UV } \\
214 \mathrm{~nm}\end{array}$ & $\begin{array}{l}\text { BGE: } 100 \mathrm{mMtris} 25 \mathrm{mM} \mathrm{CrO}_{3} \text {, } \\
25 \mathrm{mM} \mathrm{Na}_{2} \mathrm{CO}_{4} \text { and EtOH } 6 \% \\
\text { (v/v), at } \mathrm{pH} 8.2 \\
\text { Capillary: } 96 / 87 \mathrm{~cm}, 50 \mu \mathrm{m} \text { id } \\
\text { Injection: }-2 \mathrm{kV}, 50 \mathrm{~s} /-2 \mathrm{kV}, 40 \mathrm{~s} \\
\text { Separation: } 30 \mathrm{kV}, 15^{\circ} \mathrm{C}\end{array}$ & $\begin{array}{l}\text { Linear range: } \\
2-20 \mathrm{mg} \mathrm{L}^{-1} \\
\text { LOD: } 0.29-0.64 \mathrm{mg} \cdot \mathrm{L}^{-1} \\
\text { LOQ: } 2 \mathrm{mg} \mathrm{L} \mathrm{L}^{-1} \\
\mathrm{RSD}_{\text {tmig }}<1.09 \\
\mathrm{RSD}_{\text {Area }}<2.85\end{array}$ & [21] \\
\hline $\begin{array}{l}\text { Post-blast } \\
\text { samples (in } \\
\text { cash machines, } \\
\text { fireworks, } \\
\text { firecrackers) } \\
5 \text { matrices. }\end{array}$ & $\begin{array}{l}\mathrm{NH}_{4}^{+}, \mathrm{K}^{+}, \mathrm{CH}_{3} \mathrm{NH}_{3}+, \mathrm{Ca}^{2+} \\
\mathrm{Na}^{+}, \mathrm{Mg}^{2+}, \mathrm{Sr}^{2+}, \mathrm{Ba}^{2+}, \mathrm{Cs}^{+}, \\
\mathrm{Fe}^{2+}, \mathrm{Co}^{2+}, \mathrm{Mn}^{2+}, \mathrm{Ni}^{2+} \\
\mathrm{Zn}^{2+}, \mathrm{Cu}^{2+}, \mathrm{Pb}^{2+}, \mathrm{Li}^{+}\end{array}$ & $\begin{array}{l}\text { Extraction }\left(\mathrm{H}_{2} \mathrm{O} \text {, hot }\right) \text {, } \\
\text { sonication }(10 \mathrm{~min})\end{array}$ & EKC & $\begin{array}{l}\text { UV } \\
190 \mathrm{~nm}\end{array}$ & $\begin{array}{l}\text { BGE: } 15 \mathrm{mMGuan}: \mathrm{Ac}, 3 \mathrm{mM} \\
\text { 18-C-6, at pH } 4.0 \\
\text { Capillary: } 80 \mathrm{~cm}, 75 \mu \mathrm{m} \text { id } \\
\text { Injection: } 0.7 \mathrm{psi}, 4 \mathrm{~s} \\
\text { Separation: } 30 \mathrm{kV}, 20^{\circ} \mathrm{C}\end{array}$ & $\begin{array}{l}\text { Linear range: } \\
3-30 \mathrm{mg} \mathrm{L}^{-1} \\
\text { LOD: } 0.6-1.1 \mathrm{mg} \mathrm{L}^{-1} \\
\text { LOQ: } 3 \mathrm{mg} \mathrm{L}-1 \\
\mathrm{RSD}_{\text {tmig }}<2.39 \\
\mathrm{RSD}_{\text {Area }}<3.45\end{array}$ & [22] \\
\hline $\begin{array}{l}\text { Post-explosion } \\
\text { extract (1) and } \\
\text { smoke device } \\
\text { extract (1) }\end{array}$ & Frc, Glc, Lac, Suc & $\begin{array}{l}\text { Extraction }\left(\mathrm{H}_{2} \mathrm{O} \text {, hot }\right) \text {, } \\
\text { sonication }(10 \mathrm{~min})\end{array}$ & CZE & $\begin{array}{l}\text { UV } \\
270 \mathrm{~nm}\end{array}$ & $\begin{array}{l}\text { BGE: } 98 \mathrm{mMNaOH}, 120 \mathrm{mMNaCl} \text {, } \\
\text { at pH } 12.99 \\
\text { Capillary: } 60 \mathrm{~cm}, 50 \mu \mathrm{m} \text { id } \\
\text { Injection: } 0.7 \mathrm{psi}, 5 \mathrm{~s} \\
\text { Separation: }-14 \mathrm{kV}, 26.5^{\circ} \mathrm{C}\end{array}$ & $\begin{array}{l}\text { Linear range: } \\
3-30 \mathrm{mg} \mathrm{L}^{-1} \\
\text { LOD: } 5-10 \mu \mathrm{M} \\
\text { LOQ: } 3 \mathrm{mg} \mathrm{L}{ }^{-1} \\
\mathrm{RSD}_{\text {tmig }}<2.39 \\
\mathrm{RSD}_{\text {Area }}<3.45\end{array}$ & [23] \\
\hline $\begin{array}{l}\text { Samples from a } \\
\text { simulated } \\
\text { bombing attack } \\
\text { (ammonium } \\
\text { nitrate and } \\
\text { icing sugar) }\end{array}$ & $\begin{array}{l}\mathrm{Cl}^{-}, \mathrm{NO}_{2}{ }^{-}, \mathrm{NO}_{3}{ }^{-}, \mathrm{ClO}_{4}^{-}, \\
\mathrm{ClO}_{3},-\mathrm{SCN}^{-}, \mathrm{SO}_{4}^{2-}, \mathrm{CHOO}^{-}, \\
\mathrm{C}_{2} \mathrm{O}_{4}{ }^{2-}, \mathrm{PO}_{4}{ }^{3-}, \mathrm{S}_{2} \mathrm{O}_{3}{ }^{2-} \\
\mathrm{CH}_{3} \mathrm{NH}_{3}{ }^{+}, \mathrm{Sr}^{2+}, \mathrm{Ba}^{2+}, \mathrm{Li}^{+}, \\
\mathrm{K}^{+}, \mathrm{NH}_{4}^{+}, \mathrm{Na}^{+}, \mathrm{Ca}^{2+}, \mathrm{Mg}^{2+}, \\
\mathrm{Frc}, \mathrm{Glc}, \mathrm{Lac}, \mathrm{Suc}\end{array}$ & $\begin{array}{l}\text { Extraction }\left(\mathrm{H}_{2} \mathrm{O} \text {, hot }\right) \text {, } \\
\text { sonication }(10 \mathrm{~min})\end{array}$ & $\begin{array}{l}\text { CZE } \\
\text { EKC }\end{array}$ & UV & $\begin{array}{l}\text { Electrophoretic and detection } \\
\text { conditions employed in [18-20] } \\
\text { for the anions, cations and } \\
\text { carbohydrates, respectively } \\
\text { (optimized methods) }\end{array}$ & $\begin{array}{l}\text { Studied in } \\
{[18-20]}\end{array}$ & {$[24]$} \\
\hline $\begin{array}{l}\text { Residues from } \\
23 \text { CIMCs. }\end{array}$ & $\mathrm{SO}_{4}^{2-}, \mathrm{ClO}_{4}^{-}, \mathrm{ClO}_{3}^{-}, \mathrm{Cl}^{-}$ & $\begin{array}{l}\text { Solution }\left(\mathrm{H}_{2} \mathrm{O}\right) \text { and } \\
\text { sonication }(30 \text { min, } \\
\text { ambient temperature })\end{array}$ & \begin{tabular}{l}
$\qquad$ \\
$\ll$ \\
$z$ \\
0 \\
\hdashline
\end{tabular} & $\begin{array}{l}\text { UV } \\
250 \mathrm{~nm}\end{array}$ & $\begin{array}{l}\text { BGE: } 2,25 \mathrm{mM} \text { PMA, } 6,5 \\
\text { mMNaOH, } 0,75 \mathrm{mM} \text { and TEA } \\
\text { mM, at pH } 7.7 \\
\text { Capillary: } 58 \mathrm{~cm}, 50 \mu \mathrm{m} \text { id } \\
\text { Injection: } 0.5 \mathrm{psi}, 5 \mathrm{~s} \\
\text { Separation: }-30 \mathrm{kV}, 20^{\circ} \mathrm{C}\end{array}$ & Not performed & {$[25,26]$} \\
\hline $\begin{array}{l}20 \text { Samples } \\
\quad \text { (fireworks) }\end{array}$ & $\begin{array}{l}\mathrm{NO}_{3}^{-}, \mathrm{ClO}_{4}^{-}, \mathrm{ClO}_{3}^{-} \mathrm{Cl}^{-} \\
\mathrm{SO}_{4}^{2-}, \mathrm{Br}^{-}, \mathrm{S}_{2} \mathrm{O}_{3}^{2-}, \mathrm{NO}_{2} \\
\mathrm{SCN}^{-}, \mathrm{OCN}^{-}\end{array}$ & $\begin{array}{l}\text { Solution }\left(\mathrm{H}_{2} \mathrm{O}\right) \text { for } \\
\text { preblast, extraction } \\
\left(\mathrm{H}_{2} \mathrm{O}\right) \text { for postblast }\end{array}$ & $\begin{array}{l}\sum_{w} \\
> \\
Z\end{array}$ & $\begin{array}{l}\text { UV } \\
250 \mathrm{~nm}\end{array}$ & Same as $[22,23]\left(30^{\circ} \mathrm{C}\right)$ & $\begin{array}{l}\mathrm{RSD}_{\text {tmig }}<2,06 \% \\
\mathrm{RSD}_{\text {area }}<20.22 \%\end{array}$ & [27] \\
\hline Seawater & $\begin{array}{l}\text { HMX, RDX, TNB, DNB, } \\
\text { NB, TNT, 4-Am-DNT, } \\
\text { 2-Am-DNT, 2,4-DNT, } \\
\text { 2,6-DNT, 2-NT, 3-NT, } \\
\text { 4-NT }\end{array}$ & No treatment & $\stackrel{\bigcirc}{\cup} \mathrm{CZE}$ & $\begin{array}{l}\text { UV } \\
254 \mathrm{~nm}\end{array}$ & $\begin{array}{l}\text { BGE: } 10 \mathrm{mM} \text { sodium tetraborate, } \\
200 \mathrm{mM} \text { sodium cholate, } 10 \% \\
\text { (v/v) EtOH } \\
\text { Capillary: } 31.2 \mathrm{~cm}, 50 \mu \mathrm{m} \text { id } \\
\text { Injection: } 10 \mathrm{kV}, 100 \mathrm{~s} / 1 \mathrm{psi} \\
\text { Separation: } 10 \mathrm{kV}, 25^{\circ} \mathrm{C}\end{array}$ & $\begin{array}{l}\text { LOD: } \\
0.0012-0.0082 \mathrm{mg} \mathrm{L}^{-1}\end{array}$ & [28] \\
\hline $\begin{array}{l}3 \text { water samples } \\
\text { (cooking } \\
\text { plants, tap } \\
\text { water, river) }\end{array}$ & TNT, TNB, DNT, DNB & $\begin{array}{l}\text { Filtration }(0.22 \mu \mathrm{m}) \\
\text { Degasification }\end{array}$ & EKC & $\mathrm{AD}$ & $\begin{array}{l}\text { BGE: } 10 \mathrm{mM} \text { PBS, } 10 \mathrm{mM} \text { SDS, } \\
\text { at pH } 6.5 \\
\text { Working potential: }-0.7 \mathrm{~V} \\
\text { Capillary: } 55 \mathrm{~cm}, 25 \mu \mathrm{m} \text { id } \\
\text { Injection: } 17 \mathrm{kV}, 10 \mathrm{~s} \\
\text { Separation: }-17 \mathrm{kV} \text {, no T control }\end{array}$ & $\begin{array}{l}\text { Linear range: } \\
3-30 \mathrm{mg} \mathrm{L}^{-1} \\
\text { LOD: } \\
0.003-0.0047 \mathrm{mg} \mathrm{L}^{-1} \\
\mathrm{RSD}_{\text {Conc }}<5 \%(\mathrm{n}=5)\end{array}$ & [29] \\
\hline $\begin{array}{l}7 \text { Smokeless } \\
\text { gunpowders }\end{array}$ & $\mathrm{NC}$ & $\begin{array}{l}\text { Derivatization: } 2 \mu \mathrm{L} \text { of } \\
1 \times 10^{-4} \mathrm{mg} / \mathrm{mL} \text { APTS in } 15 \% \\
(\mathrm{v} / \mathrm{v}) \text { AcOH and } 2 \mu \mathrm{L} \text { of } 1.0 \mathrm{M} \\
\text { SCBH in THF to } 0.30 \mathrm{mg} \text { of } \\
\text { gunpowder } 65^{\circ} \mathrm{C} 4 \mathrm{~h} \text {. }\end{array}$ & CZE & $\begin{array}{l}\text { LIF } \\
488 \mathrm{~nm}\end{array}$ & $\begin{array}{l}\text { BGE: } 1 \mathrm{M} \text { formate, at } \mathrm{pH} 2.0 \\
\text { Capillary: } 50.2 \mathrm{~cm} \text {, } \\
50 \mu \mathrm{m} / 75.2 \mathrm{~cm}, 75 \mu \mathrm{m} \\
\text { Injection: } 0.5 \mathrm{psi}, 5 \mathrm{~s} / 15 \mathrm{~s} \\
\text { Separation: }-20 \mathrm{kV}, 25^{\circ} \mathrm{C}\end{array}$ & LOD: $12-22 \mathrm{mg} \mathrm{L}^{-1}$ & [30] \\
\hline $\begin{array}{l}64 \text { Non-explosive } \\
\text { and explosive } \\
\text { nitrocellulose } \\
\text { based samples }\end{array}$ & $\mathrm{NC}$ & $\begin{array}{l}\text { Same as [31]. Except for } \\
\text { paper samples } \\
\text { (scratching) }\end{array}$ & CZE & $\begin{array}{l}\text { LIF } \\
488 \mathrm{~nm}\end{array}$ & $\begin{array}{l}\text { BGE: } 1 \mathrm{M} \text { formate, at } \mathrm{pH} 2.0 \\
\text { Capillary: } 50.2 \mathrm{~cm}, 50 \mu \mathrm{m} \text { id } \\
\text { Injection: } 0.5 \mathrm{psi}, 5 \mathrm{~s} \\
\text { Separation: }-20 \mathrm{kV}, 25^{\circ} \mathrm{C}\end{array}$ & $\begin{array}{l}\mathrm{RSD}_{\text {electroph }}=6 \% \\
\text { for five replicates } \\
\text { and } 3 \% \text { for } \mathrm{n}=5 \\
\text { (PCA and SIMCA) }\end{array}$ & [32] \\
\hline $\begin{array}{l}\text { Cellodextrins and } \\
2 \text { NC samples }\end{array}$ & $\mathrm{NC}$ & $\begin{array}{l}\text { Depolimerization }(\mathrm{HCl}) \\
\text { Derivatization: } 5.84 \mu \mathrm{L} \text { of } \\
0.02 \mathrm{M} \text { APTS in } 15 \%(\mathrm{v} / \mathrm{v}) \\
\text { AcOH and } 1.95 \mu \mathrm{L} \text { of } 1.0 \mathrm{M} \\
\text { SCBH in THF to } 20 \mu \mathrm{L}\end{array}$ & CZE & $\begin{array}{l}\text { LIF } \\
488 \mathrm{~nm}\end{array}$ & $\begin{array}{l}\text { BGE: } 60 \mathrm{mM} \text { 6-aminocaproic } \\
\text { acid, and } 0.02 \% \mathrm{HPMC} \text { at } \mathrm{pH} 4.5 \\
\text { Capillary: } 65 \mathrm{~cm}, 50 \mu \mathrm{m} \text { id } \\
\text { Injection: } 0.7 \mathrm{psi}, 10 \mathrm{~s} \\
\text { Separation: }-30 \mathrm{kV}, 20^{\circ} \mathrm{C}\end{array}$ & $\begin{array}{l}\mathrm{RSDt}_{\text {mig }}<1.1(n=6) \\
\mathrm{RSD}_{\text {Area }}<3.5(n=6)\end{array}$ & [33] \\
\hline
\end{tabular}


Table 1 (continued)

\begin{tabular}{|c|c|c|c|c|c|c|c|c|}
\hline Sample (n) & Analyte & Sample preparation & \multicolumn{3}{|c|}{ CE mode-detection } & CE conditions & Analytical performance & Ref. \\
\hline $\begin{array}{l}\text { Starch (1 sample) } \\
\text { Nitrostarch } \\
\text { (1 sample) }\end{array}$ & $\mathrm{NC}$ & Same as [31] & 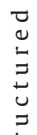 & CZE & $\begin{array}{l}\text { LIF } \\
488 \mathrm{~nm}\end{array}$ & $\begin{array}{l}\text { BGE: } 1 \mathrm{M} \text { formate, at } \mathrm{pH} 2.0 \\
\text { Capillary: } 30 \mathrm{~cm}, 50 \mu \mathrm{m} \text { id } \\
\text { Injection: } 0.5 \mathrm{psi}, 5 \mathrm{~s} \\
\text { Separation: }-20 \mathrm{kV}, 25^{\circ} \mathrm{C}\end{array}$ & Not performed & [34] \\
\hline $\begin{array}{l}\text { Dynamite } \\
\text { (4 samples) }\end{array}$ & $\mathrm{NC}$ & Same as [31] & $\begin{array}{l}5 \\
n \\
0 \\
5 \\
u \\
\Sigma\end{array}$ & CZE & $\begin{array}{l}\text { LIF } \\
488 \mathrm{~nm}\end{array}$ & $\begin{array}{l}\text { BGE: } 1 \mathrm{M} \text { formate, at } \mathrm{pH} 2.0 \\
\text { Capillary: } 31 \mathrm{~cm}, 24.5 \pm 0.9 \mu \mathrm{m} \\
\text { Injection: } 4 \text { psi, } 5 \mathrm{~s} \\
\text { Separation: }-10 \mathrm{kV}, 20^{\circ} \mathrm{C}\end{array}$ & $\begin{array}{l}\mathrm{RSD}_{\text {electroph }}=4-19 \\
\mathrm{RSD}_{\text {tmig }}<0.5 \\
\text { LOD: } 10 \mathrm{mg} \mathrm{L}^{-1}\end{array}$ & [35] \\
\hline $\begin{array}{l}\text { Residues in } \\
\text { soil from } 4 \\
\text { home-made } \\
\text { devices (IEDs } \\
\text { with inorganic } \\
\text { salts and } \\
\text { gunpowder) }\end{array}$ & 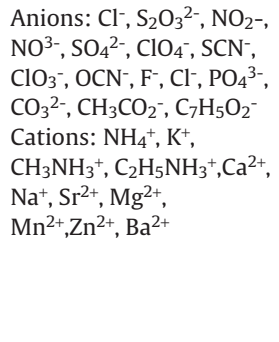 & $\begin{array}{l}\text { Swabbing } \\
\text { Solution }\left(\mathrm{H}_{2} \mathrm{O}\right) \\
\text { Sonication }(5 \mathrm{~min})\end{array}$ & & EKC & $C^{4} D$ & $\begin{array}{l}\text { Anions. BGE: } 70 \text { mMtris, } 70 \mathrm{mM} \\
\text { CHES, at pH } 8.6 \\
\text { Capillary: } 90 \mathrm{~cm}, 75 \mu \mathrm{m} \text { id } \\
\text { Injection: } 1 \mathrm{psi}, 5 \mathrm{~s} \\
\text { Separation: }-25 \mathrm{kV} \text {, no T control } \\
\text { Cations. BGE: } 10 \mathrm{mM} \text { His, } \\
50 \mathrm{mM} \text { acetic acid, } 1 \mathrm{mM} \text { HIBA, } \\
0.7 \mathrm{mM} 18-\mathrm{c}-6, \mathrm{pH} 4.2 \\
\text { Capillary: } 73 \mathrm{~cm}, 75 \mu \mathrm{m} \\
\text { Injection: } 0.1 \mathrm{psi}, 5 \mathrm{~s} \\
\text { Separation: }-25 \mathrm{kV} \text {, no T control }\end{array}$ & $\begin{array}{l}\text { Linear range: } \\
0-10 \mathrm{mg} \mathrm{L}^{-1} \\
\text { LOD: } \\
0.026-0.24 \mathrm{mg} \mathrm{L}^{-1} \\
\mathrm{RSDt}_{\mathrm{mig}}<1.14 \\
(n=10) \\
\mathrm{RSD}_{\text {area }}<13.40 \\
(n=10)\end{array}$ & [36] \\
\hline $\begin{array}{l}\text { Post-blast } \\
\text { samples (IEDs) } \\
\text { on soils }\end{array}$ & $\begin{array}{l}\mathrm{Cl}^{-}, \mathrm{NO}_{3}{ }^{-}, \mathrm{ClO}_{3}{ }^{-}, \mathrm{N}_{3}^{-}, \mathrm{F}^{-} \\
\mathrm{ClO}_{4}^{-}, \mathrm{SCN}^{-}, \mathrm{SO}_{4}{ }^{2-}, \mathrm{PO}_{4}{ }^{3-}\end{array}$ & $\begin{array}{l}\text { Extraction and } \\
\text { solution }\left(\mathrm{H}_{2} \mathrm{O}\right) \\
\text { sonication }(5 \mathrm{~min})\end{array}$ & 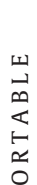 & $\mathrm{CE}$ & $C^{4} \mathrm{D}$ & $\begin{array}{l}\text { BGE: } 50 \mathrm{mMtris}, 50 \mathrm{mM} \text { CHES, } \\
0.05 \% \text { (w/v) PEI, pH } 8.6 \\
\text { Capillary: } 35 \mathrm{~cm}, 25 \mu \mathrm{m} \text { id } \\
\text { Injection: }-1 \mathrm{kV}, 1 \mathrm{~s} \\
\text { Separation: }-25 \mathrm{kV} \text {, no T control }\end{array}$ & $\begin{array}{l}\text { Linear range: } \\
0.025-3 \mathrm{mg} \mathrm{L}^{-1} \\
\text { LOD: } \\
0.018-0.079 \mathrm{mg} \mathrm{L}^{-1} \\
\mathrm{RSDt}_{\mathrm{mig}}<2.07(n=48) \\
\mathrm{RSD}_{\text {c.area }}<12.5(n=48)\end{array}$ & [37] \\
\hline $\begin{array}{l}4 \text { Consumer } \\
\text { fireworks }\end{array}$ & $\begin{array}{l}\mathrm{NH}_{4}^{+}, \mathrm{K}^{+}, \mathrm{Ca}^{2+}, \mathrm{Na}^{+}, \mathrm{Mg}^{2+}, \\
\mathrm{Sr}^{2+}, \mathrm{Ba}^{2+}, \mathrm{Cl}^{-}, \mathrm{NO}^{3-}, \mathrm{ClO}^{4-}, \\
\mathrm{ClO}_{3}{ }^{-}, \mathrm{SO}_{4}^{2-}\end{array}$ & $\begin{array}{l}\text { Solution }\left(\mathrm{H}_{2} \mathrm{O}\right) \text {, stirring } \\
(2 \mathrm{~min})\end{array}$ & $a$ & EKC & $C^{4} \mathrm{D}$ & $\begin{array}{l}\text { BGE: } 60 \mathrm{mM} \text { MES, } 2 \mathrm{mM} \\
\text { 18-c-6, at pH } 6.0 \\
\text { Capillary (x2): } 60 \mathrm{~cm} \text { (cations), } \\
67 \mathrm{~cm} \text { (anions), } 25 \mu \mathrm{m} \text { id } \\
\text { Injection: } 1 \mathrm{~min} \\
\text { Separation: }-10 \mathrm{kV} \text { (cations), } \\
20 \mathrm{kV} \text { (anions), no T control }\end{array}$ & $\begin{array}{l}\text { Linear range: } \\
1-5600 \mu \mathrm{M} \\
\text { LOD: } 1-5 \mu \mathrm{M} \\
\mathrm{RSDt}_{\mathrm{mig}}<0.4(n=9) \\
\mathrm{RSD}_{\text {area }}<7.1(n=9)\end{array}$ & [38] \\
\hline $\begin{array}{l}\text { Post blast } \\
\text { samples from } \\
8 \text { explosives } \\
\text { (Dynamite, } \\
\text { PETN, TNT, } \\
\text { RDX, PENO, } \\
\text { ANFO, V40, C4) }\end{array}$ & $\begin{array}{l}\mathrm{NH}_{4}^{+}, \mathrm{K}^{+}, \mathrm{Ca}^{2+}, \mathrm{Na}^{+}, \mathrm{Mg}^{2+}, \\
\mathrm{Cl}^{-}, \mathrm{NO}^{3-}, \mathrm{NO}^{2-}, \mathrm{SO}_{4}^{2-}, \mathrm{N}^{3-}\end{array}$ & $\begin{array}{l}\text { Solution for sand }\left(\mathrm{H}_{2} \mathrm{O}\right) \\
\text { or sawing for metal } \\
\text { and concrete }\left(\mathrm{H}_{2} \mathrm{O}\right) \text {, } \\
\text { shaking }(1 \mathrm{~min})\end{array}$ & & EKC & $C^{4} D$ & $\begin{array}{l}\text { BGE: } 20 \mathrm{mM} \text { MES, } 20 \mathrm{mM} \text { his, } \\
30 \mu \mathrm{M} \text { CTAB, } 2 \mathrm{mM} 18-\mathrm{c}-6 \text {, } \\
\text { at pH } 6.0 \\
\text { Capillary: } 50 \mathrm{~cm}, 50 \mu \mathrm{m} \text { id } \\
\text { Injection: hand pressure, } 3 \mathrm{~s} \text {. } \\
\text { Separation: }-16 \mathrm{kV} \text {, no T control }\end{array}$ & $\begin{array}{l}\text { Linear range: } \\
1-500 \mu \mathrm{M} \\
\text { LOD: } 3.7-35.7 \mu \mathrm{M} \\
\text { RSD }_{\text {area }}<9.9(n=3)\end{array}$ & [39] \\
\hline $\begin{array}{l}\text { Standard } \\
\text { solutions }\end{array}$ & EGDN, PGDN, NG, PETN & Solution in the BGE & $\begin{array}{l}\simeq \\
I \\
u \\
0 \\
\simeq \\
\simeq \\
\Sigma\end{array}$ & ECK & ED & $\begin{array}{l}\text { BGE: } 15 \mathrm{mM} \text { sodium tetraborate, } \\
20 \mathrm{mM} \text { SDS, at pH } 9.2 \\
\text { Chip: } 88 \times 17 \text { mm plexiglass, } \\
82 \text {-mm length } \\
\text { Injection: } 1.5 \mathrm{kV}, 2 \mathrm{~s} \\
\text { Separation: } 1.5 \mathrm{kV} \text {, no T control }\end{array}$ & $\begin{array}{l}\text { Linear range: } \\
10-60 \mathrm{mg} \mathrm{L}^{-1} \\
\text { LOD: } 0.3-0.5 \mathrm{mg} \mathrm{L}^{-1} \\
\mathrm{RSD}_{\text {intensities }}<2.3(n=6)\end{array}$ & [40] \\
\hline
\end{tabular}

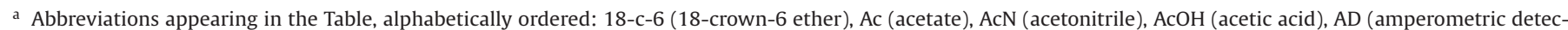

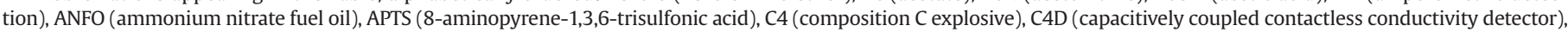

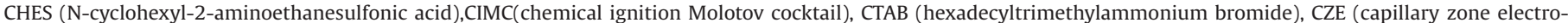

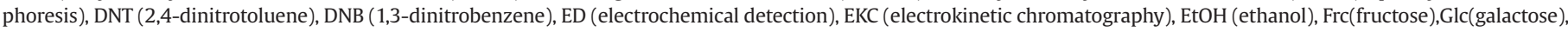

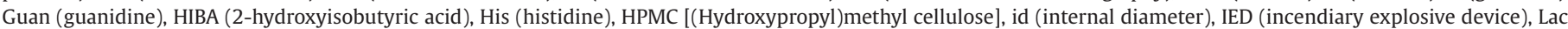

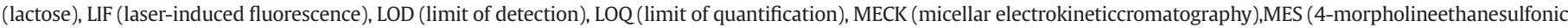

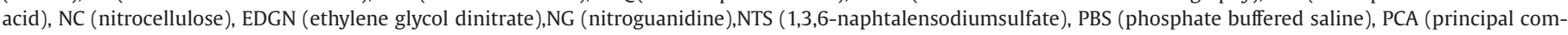

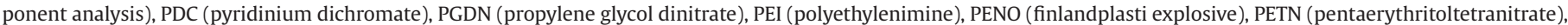

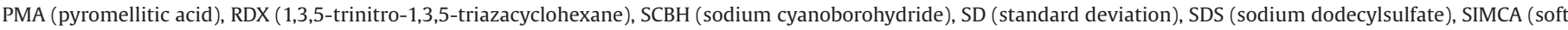

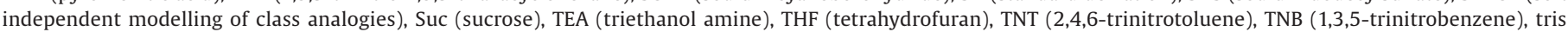
(tris(hydroxymethyl)aminomethane), UV (ultraviolet), V40 (name of a hand grenade explosive).

Other works have focused on the analysis of high-order explosives by CE due to the flexibility of this technique. Nevertheless, the aim of this research is usually the detection of explosives and their residues for environmental purposes, where electromigration techniques play an important role. Readers interested in the use of these techniques for a wide variety of environmental samples can consult recent literature. [31] Some of these works deal with the analysis of samples such as soils or water. For instance, B. Giordano et al. performed the determination of different high-order explosives (HMX, RDX, TNB, DNB, DNT and NT) from seawater and analysed directly in a CE system with UV detection at $254 \mathrm{~nm}$. In this case, the method was optimized in terms of separation medium (by modi- fying the relative amount of the surfactant in the BGE) and injection (long electrokinetic injections), and LODs between 0.07-0.8 $\mathrm{mg} \mathrm{L}^{-1}$ were achieved. [28] D. Nie et al. also attempted the determination of different high-order explosives (TNT, TNB, DNT and DNB) by CE using amperometric detection (AD) with a carbon disk electrode modified with a mesoporous carbon material. The method developed reached high selectivity, and concentrations between 0.003 and $0.005 \mathrm{mg} \mathrm{L}^{-1}$ were detected due to the efficiency of the modified carbon disk that provided enhanced electrocatalytic activity. In addition, the precision of the method was also demonstrated $($ RSDs $<5)$ by analysing real water samples from a river and a coking plant. [29] 

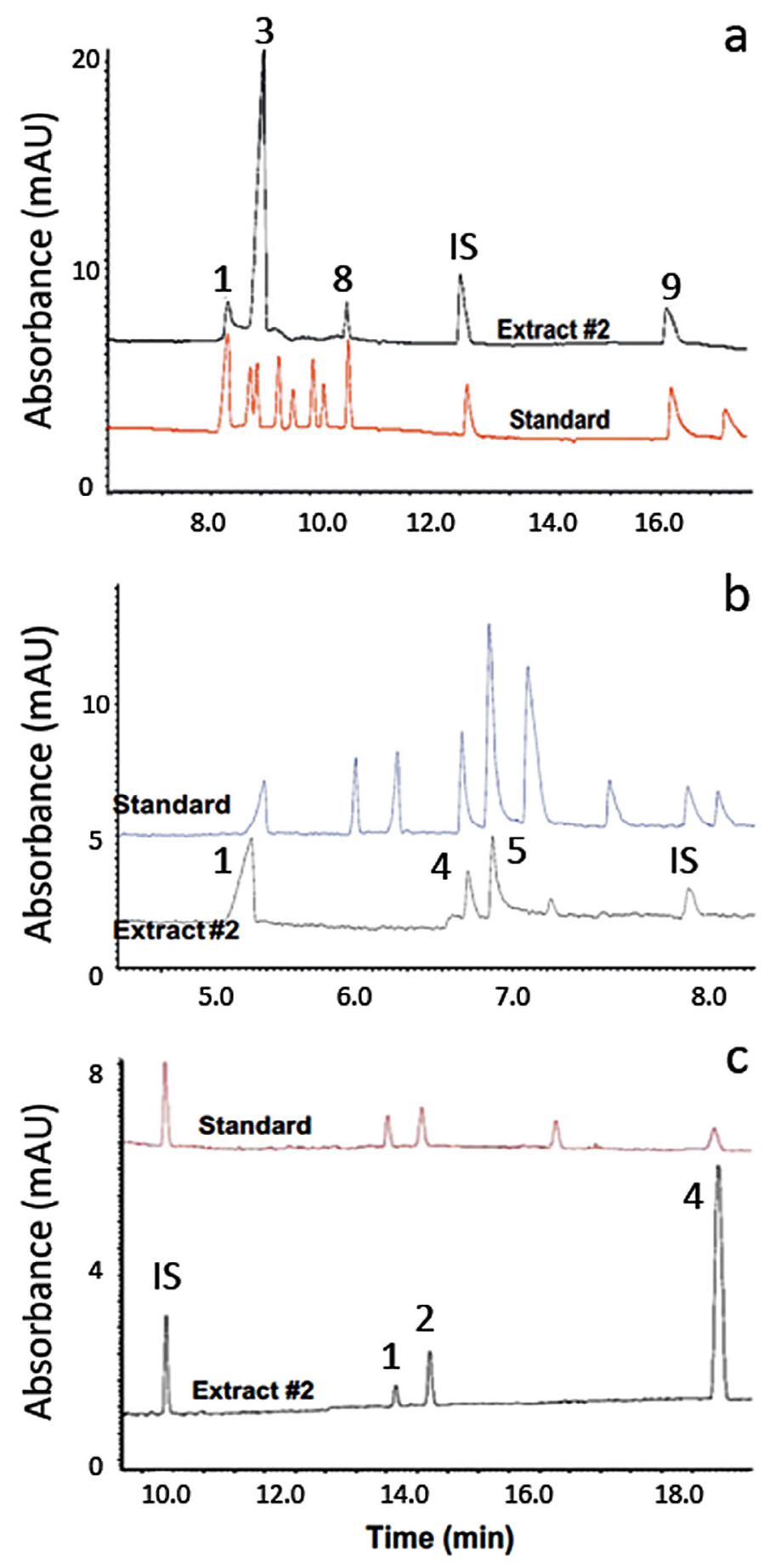

Fig. 1. CE-UV results of an aqueous extract, containing collected residues from a simulated bombing attack in a bus (home-made explosive made of ammonium nitrate and icing sugar). In a) analysis of anions (1, nitrite; 3 , thiosulfate; 8 , carbonate; IS, formate; 9 , phosphate); b) analysis of cations (1, ammonium; 4, calcium; 5 , sodium; IS, lithium; c) analysis of carbohydrates (IS, naphtalensulfonate; 1, fructose; 2, glucose, 3 , sucrose. Figures modified from [24] with permissions of Elsevier. CE conditions described in Table 1. (In colour on the web only).

Focusing on the determination of macromolecules contained in explosives such as smokeless gunpowders, M.A. Fernández de la Ossa et al. used CE with laser-induced fluorescence (LIF) detection at $488 \mathrm{~nm}$ to determine the NC content in smokeless gunpowders, before performing the derivatization process. Hence, in this case, sample preparation was more difficult compared to the studies focused on anion and cation separations. A derivatization agent was used to enhance the charge and fluorescence properties at the wavelength of the LIF detector. Pulverized gunpowders were derivatized with different agents, and finally 8-aminopyrene-1,3,6-trisulfonic acid (APTS) was considered as the most effective reagent to derivatize the sample. The method was optimized in terms of sample preparation and CE conditions; in order to enhance the selectivity of the method. The derivatized NC was detected under the detection wavelength of the LIF detector. Finally, a visual differentiation was evidenced from samples with NC with low nitrogen content $(<12 \%)$ such as collodions (non-explosive samples) and samples with NC with high nitrogen content ( $>12 \%$ ) like gunpowders (explosives), methods whose LODs were increased with stacking strategies. [30] To demonstrate the real applicability of the method, the authors analysed over 60 samples containing NC of high and low nitrogen content. The cellulose content was also analysed, in order to differentiate explosives from potential interferences at crime scene, such as cigarettes, varnishes, nail polishes or paper. After obtaining the raw electropherograms and performing different corrections (alignment and correction of the baseline), the entire electropherograms were treated (practice called fingerprinting) to carry out different statistical analyses. Principal component analysis (PCA) allowed the differentiation between samples with high and low nitrogen content. However, soft independent modelling by class analogy (SIMCA) was required to finally differentiate 12 of the 14 types of samples studied. [32] Fig. 2a shows the electropherograms with the polymeric profiles of three different samples analysed in this study (double-base gunpowder, paper and nail polish). The differences in the profiles were evident and statistically confirmed. More recently, E. Alinat et al. have modified this method, by performing an acid depolymerization prior to the derivatization process. In this method, large polymeric chains were derivatized from $\mathrm{NC}$, and the nitrogen content was quantified using an offline spectrometric technique (matrix-assisted laser desorption ionization time-of-flight mass spectrometry). [33]

Finally, M. Calcerrada et al. used the derivatization process previously described in conventional CE-LIF [30,32], but performed microstructured capillary electrophoresis (MCE) wherein the conventional separation capillary is replaced by a microstructured capillary (MSC). In the first work, two different MSCs were used in the CE equipment to carry out comparative studies with conventional capillaries of 25 - and $50-\mu \mathrm{m}$ internal diameter (id). The larger charge capacity and improved sensitivity of a six-hole MSC allowed the first determination of nitrostarch by CE-LIF. [34] Then, based on the six-hole MSC principle, the method for the determination of NC in dynamite samples was optimized in terms of sample preparation, CE conditions, capillary length, voltage and temperature. The MSC provided accurate results similar to the conventional capillaries, and traces of NC were detected in real samples. [35] Fig. 2b shows the resulting electropherogram from a diluted dynamite sample, with the NC profile. Previous studies have used multichannels in microchips or in-house CE instruments rather than commercial equipment. Nevertheless, the recent studies have demonstrated the possibility of using these microstructures as pioneering separation platform in commercial CE techniques for the analysis of complex samples. [41]

\section{Portable CE}

Table 1 summarizes the most relevant works conducted on the advancements made in the portable CE equipment during the last years. [36-39] Contrary to the reviews previously reported [11-13], we introduced P-CE independent of the conventional CE, due to the high relevance that this technology has acquired during the last years for the analysis of explosives. These developments have been achieved due to the relative simple set-up of the P-CE, compared to other separation techniques that require more complex instrumentation. 

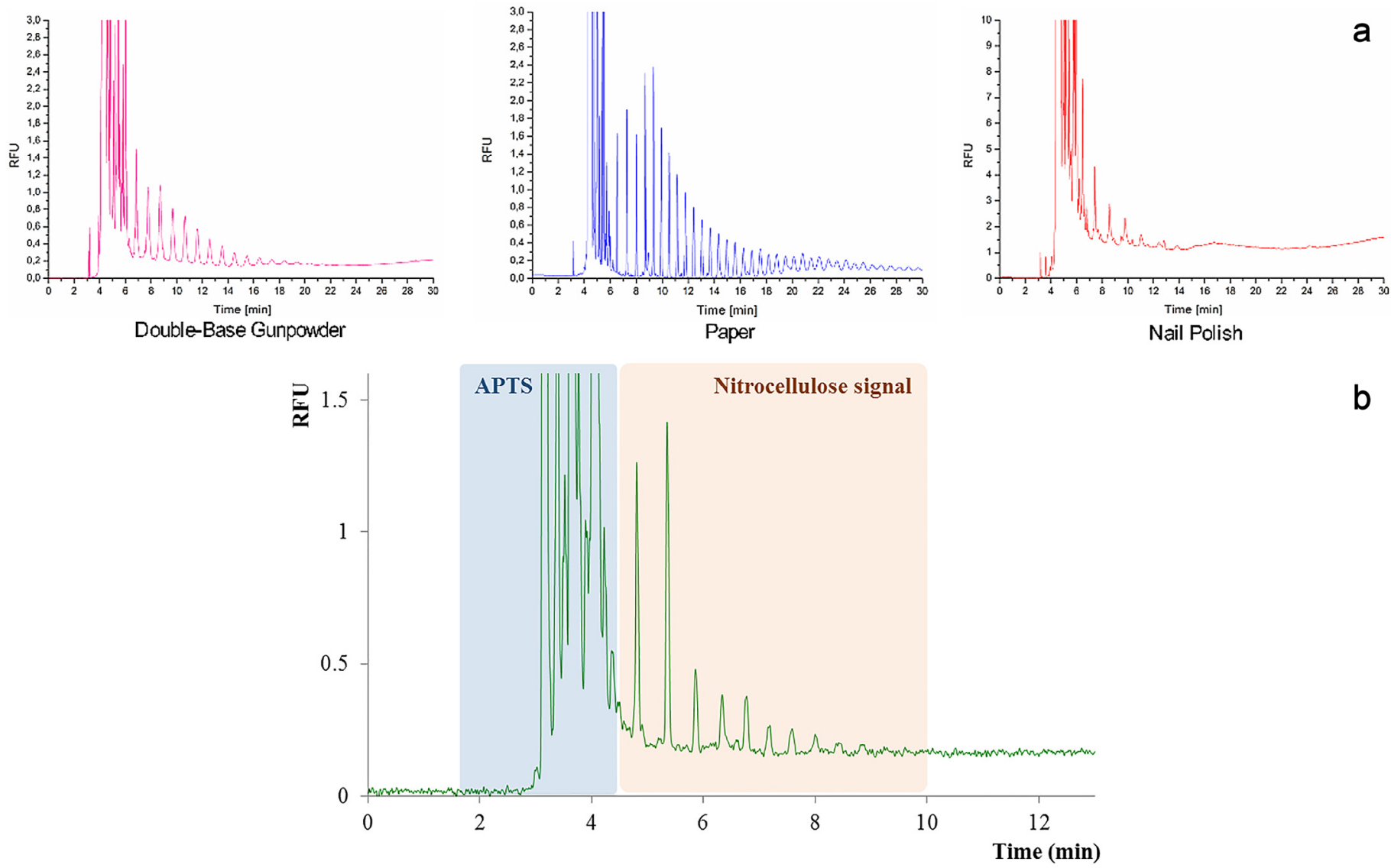

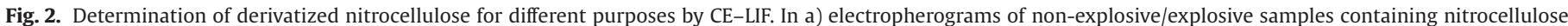

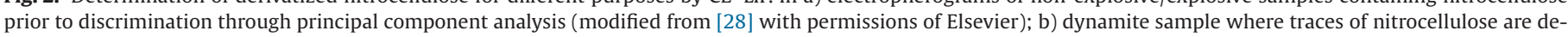
tected (taken from [32] with permissions). CE conditions described in Table 1. (In colour on the web only).

It is important to note that, contrary to the conventional CE systems, where different analytes have been determined and detection modes evaluated, P-CE has only been employed for the detection of anions and cations using capacitively coupled contactless conductivity detectors $\left(C^{4} D\right)$. Besides, none of these equipment has temperature control, which leads to less accurate results. Hence, internal standards are recommended when using this instrumentation. On the contrary, other clear advantages over conventional equipment have been demonstrated.

In 2008, J. P. Hutchinson et al. identified inorganic ions in postblast residues using a commercially available in-house modified P-CE. This pioneering approach through P-CE allowed the determination of both cations and anions from the residues. Firstly, interferences were tested in order to determine the species in the environment or added during the sample preparation. The level of contamination did not affect the interpretation of results. The authors prepared four home-made explosives using this technique, and their respective residues after detonation were analysed. Marker ions obtained from the formulations of the explosives are in agreement with the results. Besides, a comparison with an IC method was performed, and despite obtaining higher LODs for the P-CE, results were in excellent agreement. Finally, a rapid analysis ( $<45 \mathrm{~s})$ method was proposed for anions, involving elimination of some of the ions (not usually markers of the explosives), and reduction of the capillary length. In spite of the fast separation, the authors recommended a baseline resolution to avoid false-positive or false-negative results. [36]
More recently, G. A. Blanco et al. proposed the identification of anions from IEDs using sequential injection CE (SI-CE). In SI-CE, the sample and BGE are alternatively flowed through the interface under computer-controlled conditions of flow and voltage, thus allowing fine control on the injection conditions. Fig. 3a depicts a scheme of the equipment used. The SI-CE instrument consisted of a double syringe pump used to deliver sample and BGE through the system. A two-position injector valve was employed to alternately deliver the sample or BGE to the interface. After selecting the ions of interest, the authors optimized the method to achieve good separation. The selectivity of inorganic anions in CE was modified by the introduction of an ion-exchange component to the electrophoretic separation, which is known as ion-exchange electrokinetic chromatography. Poly(ethylenimine)was added to the BGE in order to separate the ions, although poor reproducibility (RSD of $5 \%$ for $n=6$ ) was obtained in migration times, which is not acceptable for identification purposes. Nevertheless, an IS was employed to perform electropherogram treatments and enhance the reproducibility of the method. After selecting the BGE, the hardware was optimized by selecting a capillary and an injection in order to obtain excellent LODs and a proper separation efficiency in terms of peak resolution. Besides, the method allowed highly reproducible analysis as can be seen in Table 1. Various anions were successfully detected, as can be seen in Fig. 3b [37], upon analysis of the residues of an in-house-made explosive post detonation.

J. Sáiz et al. fabricated a dual P-CE for the concurrent determination of anions and cations, and applied it to the detection of these 


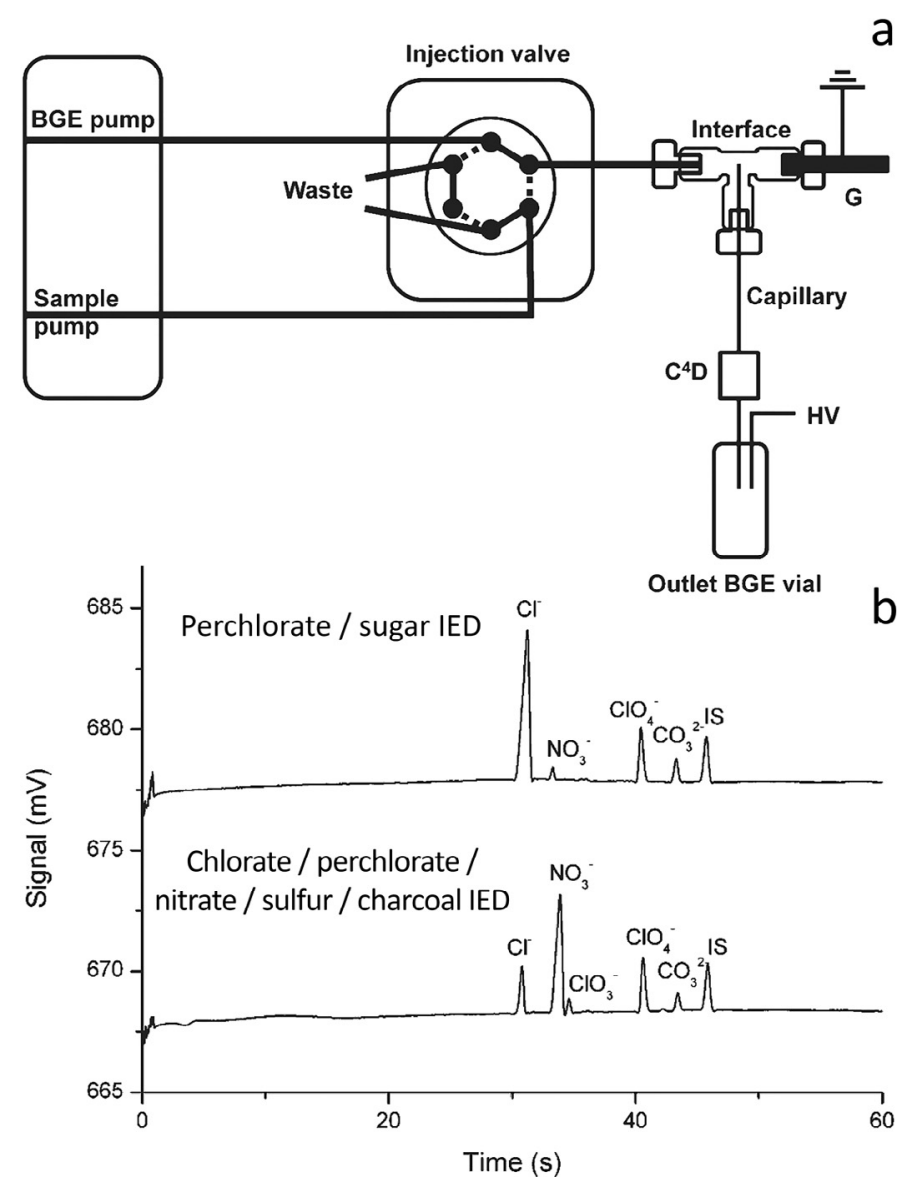

Fig. 3. Determination of different cations and anions using sequential injection in P-CE-C $C^{4} \mathrm{D}$. In a) prototype with sequential injection for the identification of two IEDs; in b) resulting electropherograms of two IEDs. CE conditions described in Table 1(taken from [35] with permissions of ACS). (In colour on the web only).

species in consumer fireworks. Contrary to the previously used P-CE equipment, this one comprised two different capillaries, with different lengths in order to perform the separation of anions and cations separately. Different voltages were also needed for each capillary. However, the system required the same BGE for the two parallel separations. Fig. 4a depicts a scheme of the dual P-CE. The BGE was first selected based on its ability to separate both anions and cations. The identification of ions was performed by spiking the samples with the ions of interest, as temperature control and IS were not used. Figures of merit of this method indicated the possibility of increasing the voltage during the separation to detect lowmobility ions, or increasing the injection time to detect ions at very low concentrations. Finally, the authors analysed real fireworks (fuses and charges) and apart from the ions expected in the electropherogram (as a known sample composition is used), other ions were also identified, due to interferences from the matrix, or irregularities in the manufacturing process. Fig. $4 \mathrm{~b}$ shows the electropherograms of one of the samples. [38] Thanks to the dual detection technique, fireworks were for the first time analysed for the detection of cations and anions, providing more complete information than the previous works, where only anions were determined. [27]

Finally, E. G. Kobrinet al. used a custom-made P-CE equipment to detect residues from different explosives. Contrary to the previous works, sample introduction strategy was in this case performed by the dual opposite end injection (first the anions and then the cations). Two IS were employed to obtain more precise results. By studying different matrices, different anions from the explosives were detected. Ten cations and anions were detected in $<4 \mathrm{~min}$. In ad- dition, the entire electropherograms from the samples (profiling) were taken to carry out PCA analysis, and the identification of specific explosives was achieved. [39]

\section{Microchip CE and CE sensors}

This section deals with microchip CE, a miniature form of CE. Surprisingly, despite being a promising technology, scarce research has been published during the period from 2008 to March 2005, compared to that reported during the previous years. [11-13,42]

As shown in Table 1, only a single study has been conducted on analysis of explosives using the microchip CE. E. Piccin et al. used a microchip for sensitive and rapid measurements of the nitrate ester explosives. Despite obtaining low sensitivity for PETN, detection of analytes at the pg level was shown to be possible using a microchip, in an assay lasting for $160 \mathrm{~s}$. Fig. 5a shows the results from the studies on a mixture of explosives. A series of analysis of the standard mixtures conducted showed enhanced reproducibility (Fig. 5b). [40] However, this microchip was not tested in real postblast or pre-blast samples, which could be relevant for the implementation of this technology in an extended way and useful for studying the speed, sensitivity, efficiency, portability, cost and sample size compared to conventional CE. New patents have proposed the use of portable and simple devices, which could be considered as chemical sensors, to overcome fast analysis of explosives, for example, a mobile app electrophoresis analysis system that provides chemical information [43] Another example is the invention of a device for detecting the presence of a hazardous target molecule in a sample, such as biological agents, toxic chemicals, poisonous gases, narcotics or traces of explosive material or residues, and where an electrophoretic or dielectrophoretic field gradient between the sample and the substrate is required. [44]

\section{Comparison of $\mathrm{CE}$ with other emerging techniques}

The applicability of CE and its diverse types in the analysis of explosives and their residues has been discussed earlier. In addition, note that the classical analysis of explosives by $\mathrm{CE}$ includes the use of IC as an ideal orthogonal separation technique to complement and/or confirm the results obtained by CE. [6] Nevertheless, it is important to briefly mention the results obtained during the recent years using other emerging techniques for the analysis of explosives. Raman spectroscopy and mass spectrometry coupled with other separation and spectroscopic techniques have been used in this field. However, only specific examples of other techniques have been included to compare the strengths and weaknesses of these techniques over $\mathrm{CE}$.

A recent review focused on the use of Raman spectroscopy for the analysis of explosives has been reported. [2] This technique possesses some advantages which make it ideal for explosive analysis. On the one hand, samples can be analysed directly in the equipment, thus making sample preparation easier compared to other separation techniques. For example, M. López-López et al. analysed for the first time dynamite using Raman spectroscopy, and some of its major components (ammonium nitrate, ethylene glycol dinitrate and sawdust) were successfully detected without any sample treatment. To perform this analysis, Raman mapping was carried out to create chemical images which allowed the differentiation among these components in the heterogeneous sample through the Raman spectra.45 However, for the detection of nitrocellulose, a minor compound in dynamites, sample treatment based on the flocculation of this substance was previously required. The determination of nitrocellulose in dynamite was also achieved by $\mathrm{CE}$, where sample derivatization was required for the detection at pg levels of nitrocellulose contained in this sample. [35] If surfaceenhanced Raman spectroscopy (SERS) is used to enhance the 

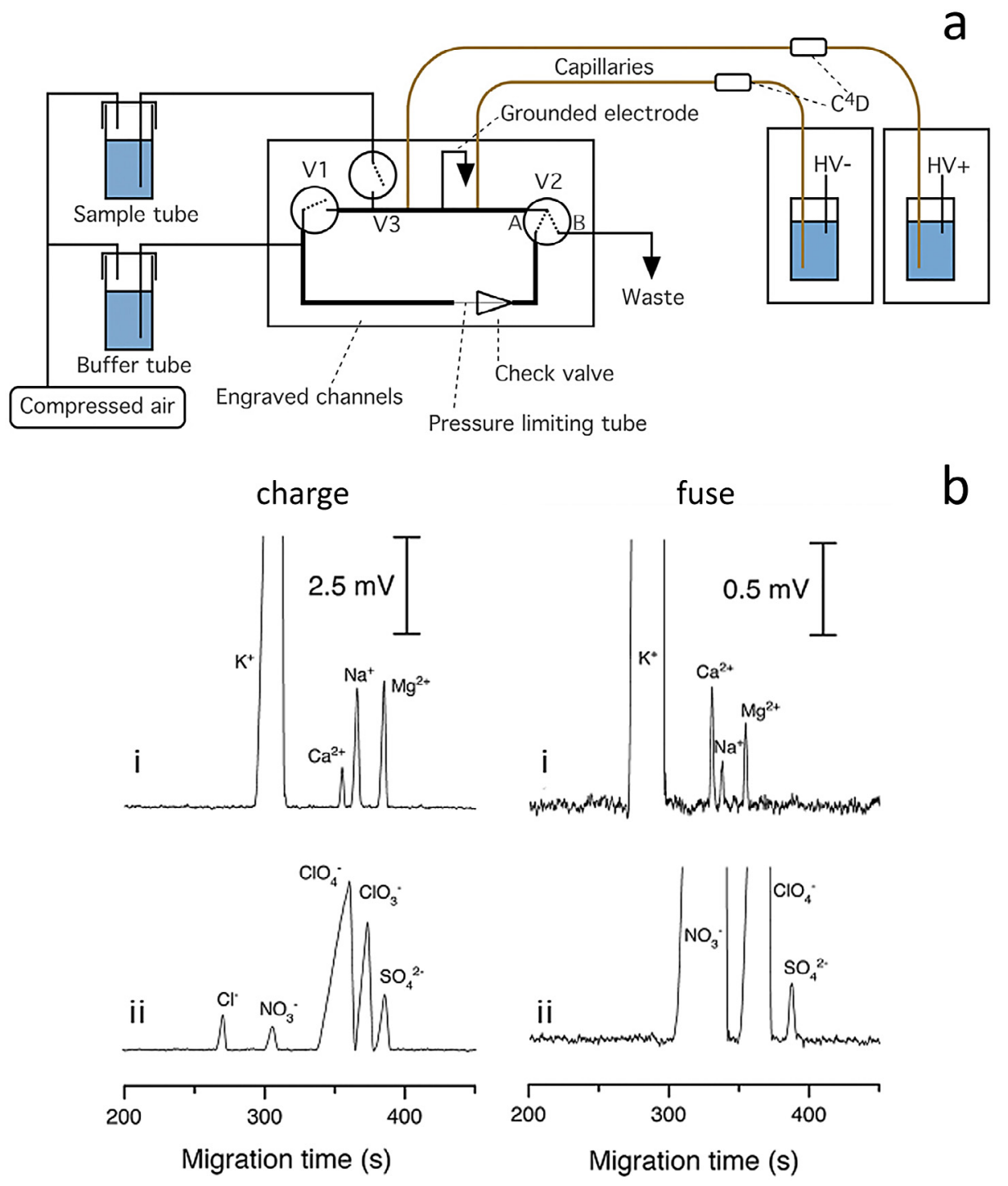

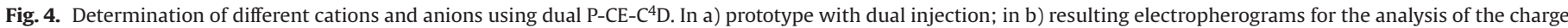
and fuse of a consumer firecracker. CE conditions described in Table 1. (Modified from [41] with permissions of Elsevier). (In colour on the web only).

spectrum signal, sample preparation becomes more tedious, not only because of the addition of metal nanoparticles, an additional step in the procedure, but also due to the irreproducibility problems that SERS present compared to other techniques. However, some results are promising for further investigation. For example, S. Botti et al.
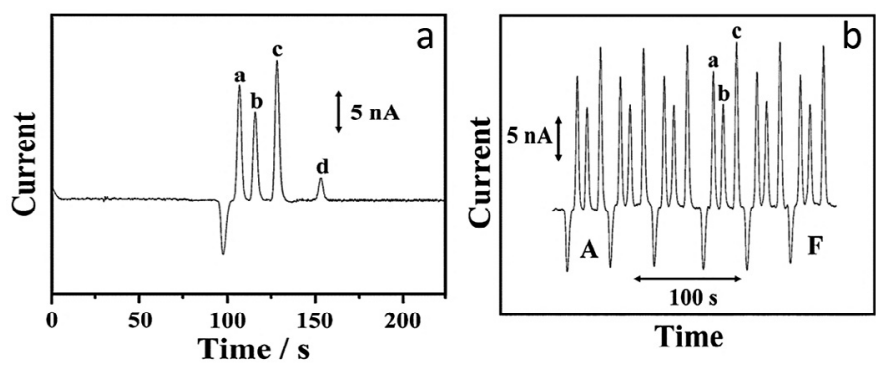

Fig. 5. Microchip CE-ED for the analysis of nitrate ester explosives. In a) the resulting electropherogram of a standard mixture (a, ethylene glycol dinitrate, b, propylene glycol dinitrate; c, nitroglycerin; d, pentaerythritoltetranitrate); in b) a reproducibility study of the CE microchip (a,b and c as in Fig. 5a). Figure taken from [38] with permissions of RSC. CE conditions in Table 1. (In colour on the web only). applied SERS with commercial substrates such as klarite ${ }^{\circledR}$, to detect PETN, EDGN, RDX and TNT at concentration levels of tens of pg. [45] Another advantage of Raman spectroscopy is its potential to identify compounds in a sample (fingerprinting), as Raman spectra are unique for each compound. $[45,46]$ Nevertheless, when samples are composed of homogeneous mixtures, a separation technique is usually more useful, as this analysis allows a separation of the components prior to detection and confirmation with other techniques or validated methods. [24] Besides, quantification of analytes is a difficult task when employing Raman spectroscopy; thus, this technique is not usually considered for environmental applications where quantification can be required, and is used primarily in the detection of potential compounds for security purposes or in forensic casework. In summary, the advantages of Raman spectroscopy depend on short duration of analysis and easier sample preparation compared to the separation or other spectrometric techniques; the miniaturized format of this technique has been used for in situ analysis. [45]

MS has been established as a powerful analytical technique in many analytical fields and more specifically in forensic chemistry, due to its potential to unequivocally detect the compounds present in a sample and also its quantification capability at trace levels and 
with high precision. For example, desorption electrospray ionization mass spectrometry (DESI-MS) has been extensively used in the analysis of high-order explosives such as TNT and HMX, with the detection levels on the pg and fg scales, thus being ideal for trace analysis. [47] MS can also be coupled other techniques in order to improve the analysis performance. For example, HPLC-MS can be used to separate and subsequently unequivocally detect analytes present in the samples. A. L. Russell et al. applied this technique to determine the composition of intensive munitions explosives (IMX). Two of them, which contained 2,4-dinitroanisole (DNAN), 1,2,4triazon-5-one (NTO), 1-nitroguanidine (NQ) and RDX were subjected to analysis, reaching detection limits ranging from 0.007 to $0.009 \mathrm{mg} \mathrm{L}^{-1}$ [48] Finally, note that CE coupled with MS techniques provides additional selectivity compared to CE with DAD detection and allows the confirmation of the detected molecules. This hyphenated technique has been used in the forensic analysis of some samples such as drugs (amphetamine, methadone and morphine) [49], and also in metabolomics. [50] Nevertheless, it has limited application in the analysis of explosives and their residues [49] and no studies have been published during the reviewed period.

Ion mobility spectrometry (IMS) is another emerging technique for the analysis of explosives and other forensic evidence. This technique, coupled with MS, has recently proven to be very efficient for the analysis of high-order explosives. J.

Lee et al. used corona discharge ionization combined with IMSMS to analyse RDX, TNT, PETN, HMX and DNT. The LODs ranged between $0.1 \mathrm{ng}$ for RDX and $10 \mathrm{ng}$ for DNT, thus demonstrating its potential for trace analysis. [51] Consequently, the use of MS or its coupling to separation techniques provides an improved sensitivity and confirmation of the molecules detected, at the expense of more expensive and complex equipment and limitations regarding the portable formats compared to CE.

Finally, it is important to highlight the applications of nonelectrophoretic miniaturised devices in the last years. [52] For example, A. Choodum et al. proposed the use of an iPhone as a novel platform for the development of a rapid and on-site semiquantitative analysis of TNT in soil samples, after a colourimetric test. Images were captured by the camera in the device after the colourimetric test, and calibration curves between the RGB values and the concentration of the colourimetric product were obtained, with acceptable relative errors (RSDs between $0.4 \%$ and $6.3 \%$ ) and good precision (RSDs between $2.09 \%$ and $7.43 \%$ ). [53] In addition, fluorescent sensors seem to be promising devices for the detection of nitroaromatic explosives. Y. Gong et al. created a thriphenylene-2,6,10-tricarboxylate and $\mathrm{Tb}^{3+}$ building block to perform the selective sensing of TNT, TNP, 2,4-DNT, 2,6-DNT and nitrobenzene (NB). Apart from conducting a reproducible analysis for at least five times before regenerating the block, the device was able to detect solutions of those explosives at $5 \mathrm{mg} \mathrm{L}^{-1}$. In another approach, S. Zhang et al. developed other metal-organic frameworks called NENU-503 (composed of $\left[\mathrm{Cd}_{2} \mathrm{Cl}\left(\mathrm{H}_{2} \mathrm{O}\right)(\mathrm{L})\right] \cdot 4.5 \mathrm{~N}, \mathrm{~N}-$ dimethylacetamide) to selectively detect nitroaromatic groups, and differentiate NB, 1,3-DNB and TNP with different nitro groups by the shift of the peak spectra. [54] From the above-mentioned results, it is clear that sensing devices are currently emerging as promising candidates for fast and portable equipment, and also of great interest in the in situ analysis for security homeland. However, these sensors are not well established compared to the conventional analytical techniques such as CE.

In summary, the advantages of Raman spectroscopy, MS and sensing devices make them ideal candidates for the analysis of explosives. Considering some recommendations for the analysis of explosives [8,9], it is evidenced that results obtained from these techniques are needed as complementary or confirmatory analysis obtained from CE and IC, with the aim of obtaining reliable results, precision and capability for trace analysis of explosives.

\section{Conclusions and perspectives}

In general terms, recent research has demonstrated the potential of CE for the separation, identification and often quantification of the specific species of interest for the analysis of explosives. In fact, regarding the samples, a wide variety of commercial and military explosives has been studied through this technique, obtaining promising results (in terms of separation efficiency, cost, analysis time and LODs).

It is evident that the conventional CE still plays an important role in the analysis of explosives, as it is the most established and robust instrumentation technique. In addition, new methodological approaches and instrumentation developments have supplied some requirements of explosive analysis. Statistical treatments are also being used to facilitate the interpretation of results. This indicates that conventional CE is still an interesting field for the developments in the analysis of explosives.

$\mathrm{P}-\mathrm{CE}$ is a candidate for the in situ analysis of explosives at crime scenes or in environmental monitoring areas as more than acceptable results, compared to the conventional $\mathrm{CE}$, have been obtained through this emerging instrumentation technique. Further studies must be conducted on P-CE, owing to its high versatility and also simple set-up compared to other conventional CE techniques. For example, the problems of reproducibility may be addressed in order to obtain more precise results. Besides, other detection modes have not been optimized, and the only a few explosives have been analysed using this equipment.

Finally, during the reviewed period (from the beginning 2008 to March 2015), very limited studies have been conducted on the application of microchip technology in the analysis of explosives, though discussed in previously published reviews. However, recent inventions reveal that the ideas and goals of these technologies are still of great interest.

Future investigations should be focused on the development of improved CE instrumental set-ups, including the miniaturised formats, with higher selectivity, more robust equipment and also higher sensitivities, in for the effective analysis of explosives.

\section{Acknowledgements}

M. Calcerrada thanks the Ministry of Education (FPU12/00991) for his predoctoral grant. Help provided by Ignacio Jimeno for image editing is gratefully acknowledged.

\section{References}

[1] J.P. Agrawal, High Energy Materials. Propellants, Explosives and Pyrotechnics, Wiley, Hoboken, 2010.

[2] M. López-López, C. García-Ruiz, Infrared and Raman spectroscopy techniques applied to identification of explosives, TrAC Trends Anal. Chem. 54 (2014) 36-44.

[3] B.J. Buchanan, Gunpowders, explosives and the State, Ashgate Publishing, Farnham, 2006.

[4] E. Dabek-Zlotorzynska, V. Celo, M.M. Yassine, Recent advances in CE and CEC of pollutants, Electrophoresis 29 (2008) 310-323.

[5] J. Yinon, Explosives, in: Handbook of Analytical Separations, vol. 6, 2008, pp. $823-838$.

[6] L. Barron, E. Gilchrist, Ion chromatography-mass spectrometry: a review of recent technologies and applications in forensic and environmental explosives analysis, Anal. Chim. Acta 806 (2014) 27-54.

[7] EPA Method 8330B, Nitroaromatics, nitramines, and nitrate esters by high performance liquid chromatography (hplc). < http://www.epa.gov/solidwaste/ hazard/testmethods/pdfs/8330b.pdf>, 2006 (accessed 06.2015)

[8] Technical/Scientific Working Group for Fire and Explosions Analysis (T/SWGFEX), Recommended Guidelines for Forensic Identification of Intact Explosives. $<$ https://cdn.preterhuman.net/texts/terrorism_and_pyrotechnics/explosives/ Detection/Identification\%20of\%20Intact\%20Explosives.pdf>, 2009 (accessed 11.2015).

[9] Technical/Scientific Working Group for Fire and Explosions Analysis (T/SWGFEX), Recommended Guidelines for Forensic Identification of Post-Blast Explosive Residues. <http://media.wix.com/ugd/4344b0_ae0bb0bf60684aa488c0f1 d01add3c06.doc?dn=TWGFEX_POST_BLAST_8Sept13_2009.doc >, 2009 (accessed 11.2015). 
[10] M. Szumski, Equipment, in: B. Buszewski, E. Dziubakiewicz, M. Szumski (Editors), Electromigration Techniques, Theory and Practice, Springer, Berlin, 2013.

[11] M. Pumera, Analysis of explosives via microchip electrophoresis and conventional capillary electrophoresis: a review, Electrophoresis 27 (2006) 244-256.

[12] M. Pumera, Trends in analysis of explosives by microchip electrophoresis and conventional CE, Electrophoresis 29 (2008) 269-273.

[13] C. Sarazin, N. Delaunay, A. Varenne, C. Costanza, V. Eudes, P. Gareil, Capillary and microchip electrophoretic analyses of explosives and their residues, Sep. Pur. Rev. 39 (2010) 63-94

[14] C.D. Garcia, K.Y. Chumbimuni-Torres, E. Carrilho, Capillary Electrophoresis and Microchip Capillary Electrophoresis: Principles, Applications, and Limitations, John Wiley \& Sons, Inc., New Jersey, 2013.

[15] K. Szomborg, F. Jongekrijg, E. Gilchrist, T. Webb, D. Wood, L. Barron, Residues from low-order energetic materials: the comparative performance of a range of sampling approaches prior to analysis by ion chromatography, Forensic Sci. Int. 233 (2013) 55-62.

[16] C. Love, E. Gilchrist, N. Smith, L. Barron, Detection of anionic energetic material residues in enhanced fingermarks on porous and non-porous surfaces using ion chromatography, Forensic Sci. Int. 231 (2013) 150-156.

[17] D. Gaurav, A.K. Malik, P.K. Rai, High-performance liquid chromatographic methods for the analysis of explosives, Crit. Rev. Anal. Chem. 37 (2007) 227-268.

[18] E.S. Gilchrist, P.N. Nesterenko, N.W. Smith, L.P. Barron, Organic solvent and temperature-enhanced ion chromatography-high resolution mass spectrometry for the determination of low molecular weight organic and inorganic anions, Anal. Chim. Acta 865 (2015) 83-91.

[19] K.G. Lahoda, O.L. Collin, J.A.H.E. LeClair, S.H. Wise, B.R. McCord, A survey of background levels of explosives, and related compounds in the environment, J. Forensic Sci. 53 (2008) 802-806.

[20] C. Sarazin, N. Delaunay, A. Varenne, C. Costanza, V. Eudes, P. Gareil, Simultaneous capillary electrophoresis analysis of inorganic anions and cations in post-blast extracts of acid-aluminium mixtures, J. Sep. Sci. 33 (2010) 3177-3183.

[21] C. Sarazin, N. Delaunay, A. Varenne, J. Vial, C. Costanza, V. Eudes, et al., Identification and determination of inorganic anions in real extracts from preand post-blast residues by capillary electrophoresis, J. Chromatogr. A 1217 (2010) 6971-6978.

[22] C. Sarazin, N. Delaunay, C. Costanza, V. Eudes, P. Gareil, Capillary electrophoresis analysis of inorganic cations in post-blast residue extracts applying a guanidinium-based electrolyte and bilayer-coated capillaries, Electrophoresis 33 (2011) 1828-1891.

[23] C. Sarazin, N. Delaunay, C. Costanza, V. Eudes, P. Gareil, Application of a new capillary electrophoretic method for the determination of carbohydrates in forensic, pharmaceutical, and beverage samples, Talanta 99 (2012) 202-206.

[24] C. Sarazin, N. Delaunay, C. Costanza, V. Eudes, P. Gareil, On the use of capillary electrophoresis for the determination of inorganic anions and cations, and carbohydrates in residues collected after a simulated suicide bombing attack, Talanta 103 (2013) 301-305.

[25] C. Martín-Alberca, J.L. Ferrando, C. García-Ruiz, Anion markers for the forensic identification of chemical ignition Molotov cocktail composition, Sci. Justice 53 (2013) 49-54.

[26] C. Martín-Alberca, J. Sáiz, J.L. Ferrando, C. García-Ruiz, Qualitative determination of inorganic anions in incendiary device residues by capillary electrophoresis, Anal. Methods 4 (2012) 2680-2686

[27] C. Martín-Alberca, M.A. Fernández de la Ossa, J. Saiz, J.L. Ferrando, C. García-Ruis, Anions in pre- and post-blast consumer fireworks by capillary electrophoresis, Electrophoresis 35 (2014) 3272-3280.

[28] B.C. Giordano, D.S. Burgi, G.E. Collins, Direct injection of seawater for the analysis of nitroaromatic explosives and their degradation products by micellar electrokinetic cromatography, J. Chromatrogr. A 1217 (2010) 4487-4493.

[29] D. Nie, P. Li, D. Zhang, T. Zhou, Y. Liang, G. Shi, Simultaneous determination of nitroaromatic compounds in water using capillary electrophoresis with amperometric detection on an electrode modified with mesoporous nanostructured carbon material, Electrophoresis 31 (2010) 2981-2988.

[30] M.A. Fernández de la Ossa, M. Torre, C. García-Ruiz, Determination of nitrocellulose by capillary electrophoresis with laser-induced fluorescence detection, Anal. Chim. Acta 745 (2012) 149-155.

[31] E. Bald, P. Kubalczyk, S. Studzińska, E. Dziubakiewicz, B. Buszewski, Application of electromigration techniques in environmental analysis, in: B. Buszewski (Editor), Electromigration Techniques, Springer-Verlag, Berlin, 2013, pp. 335-353.
[32] M.A. Fernández de la Ossa, F. Ortega-Ojeda, C. García-Ruiz, Determination of non-explosive and explosive samples through nitrocellulose fingerprints obtained by capillary electrophoresis, J. Chromatogr. A 1302 (2013) 197-204.

[33] E. Alinat, N. Delaunay, C. Przybylski, R. Daniel, X. Archer, P. Gariel, Capillary electrophoresis fingerprinting of 8-aminopyrene-1,3,6-trisulfonate derivatized nitrocellulose after partial acid depolymerisation, J. Chromatogr. A 1387 (2015) 134-143.

[34] M. Calcerrada, M.A. Fernández de la Ossa, P. Roy, M. González-Herráez, C. García Ruiz, Fundamentals on new capillaries inspired by photonic crystal fibers as optofluidic separation systems in CE, Electrophoresis 36 (2015) 433440.

[35] M. Calcerrada, M.A. Fernández de la Ossa, P. Roy, M. González-Herráez, C. García Ruiz, A microstructured-capillary electrophoresis method for dynamite analysis, Submitted, 2015

[36] J.P. Hutchinson, C. Johns, M.C. Breadmore, E.F. Hilder, R.M. Guijt, C. Lennard, et al., Identification of inorganic ions in post-blast explosive residues using portable CE instrumentation and capacitively coupled contactless conductivity detection, Electrophoresis 29 (2008) 4593-4602.

[37] G.A. Blanco, Y.H. Nai, E.F. Hilder, R.A. Shellie, G.W. Dicinoski, P.R. Haddad, et al., Identification of inorganic improvised explosive devices using sequential injection capillary electrophoresis and contactless conductivity detection, Anal. Chem. 83 (2011) 9068-9075.

[38] J. Sáiz, M.T. Duc, I.J. Koenka, C. Martín-Alberca, P.C. Hauser, C. García-Ruiz, Concurrent determination of anions and cations in consumer fireworks with a portable dual-capillary electrophoresis system, J. Chromatogr. A 1372 (2014) $245-252$.

[39] E.-G. Kobrin, H. Lees, M. Fomitšenko, P. Kubáň, M. Kaljurand, Fingerprinting postblast explosive residues by portable capillary electrophoresis with contactless conductivity detection, Electrophoresis 35 (2015) 1165-1172.

[40] E. Piccin, N. Dossi, A. Cagan, E. Carrilho, J. Wang, Rapid and sensitive measurements of nitrate ester explosives using microchip electrophoresis with electrochemical detection, Analyst 134 (2009) 528-532.

[41] Y. Sun, Y.C. Kwok, N.T. Nguyen, Faster and improved microchip electrophoresis using a capillary bundle, Electrophoresis 28 (2007) 4765-4768.

[42] J. Wang, Electrochemical sensing of explosives, Electroanalysis 19 (2007) 415-423.

[43] G. Klunder, C.R. Cooper, J.H. Satcher Jr., E.A. Tekle, US patent Application Publication, Mobile app for chemical detection, No. US 2014/0080129 A1, March 2014.

[44] C.A. Frayling, B.F. Nogueira De Sousa Soares, Nanoporeplasmonic detector, Patent No. WO/2014/167321, October 2014.

[45] S. Botti, S. Almaviva, L. Cantarini, A. Palucci, A. Puiu, A. Rufoloni, Trace level detection and identification of nitro-based explosives by surface-enhanced Raman spectroscopy, J. Raman Spectrosc. 44 (2013) 463-468.

[46] M. López-López, J.L. Ferrando, C. García-Ruiz, Dynamite analysis by Raman spectroscopy as a unique analytical tool, Anal. Chem. 85 (2013) 2595-2600.

[47] M. Morelato, A. Beavis, P. Kirkbride, C. Roux, Forensic applications of desorption electrospray ionisation mass spectrometry, Forensic Sci. Int. 226 (2013) 1021.

[48] A.L. Russell, J.M. Seiter, J.G. Coleman, B. Winstead, A.J. Bednar, Analysis of munitions constituents in IMX formulations by HPLC and HPLC-MS, Talanta 128 (2014) 524-530.

[49] K. Klepárník, Recent advances in the combination of capillary electrophoresis with mass spectrometry: from element to signle-cell analysis, Electrophoresis 34 (2013) 70-85.

[50] R. Ramautar, G.W. Somsen, G.J. de Jong, CE-MS for metabolomics: developmentsand applications in the period 2012-2014, Electrophoresis 36 (2015) 212-224.

[51] J. Lee, S. Park, S.G. Cho, E.M. Goh, S. Lee, S. Koh, et al., Analysis of explosives using corona discharge ionization combined with ion mobility spectrometrymass spectrometry, Talanta 120 (2014) 64-70.

[52] A.A. Adams, P.T. Charles, A.R. Deschamps, A.W. Kusterbeck, Demonstration of submersible high-throughput microfluidic immunosensors for underwater explosives detection, Anal. Chem. 83 (2011) 8411-8419.

[53] A. Choodum, P. Kanatharana, W. Wongniramaikul, N.N. Daeid, Using the iPhone as a device for a rapid quantitative analysis of trinitrotoluene in soil, Talanta 115 (2013) 143-149.

[54] S. Zhang, D. Du, J. Qin, S. Bao, S. Li, W. He, et al., A fluorescent sensor for highly selective detection of nitroaromatic explosives based on a 2D, estremely stable, metal-organic framework, Chem. Eur. J. 20 (2014) 3589-3594. 